\title{
Feed-forward observer-based intermittent fault detection
}

\author{
T. Sedighi ${ }^{\text {a,* }}$, P.D. Foote ${ }^{\mathrm{b}}$, P. Sydor ${ }^{\mathrm{a}}$ \\ a EPSRC Centre in Through-Life Engineering Services, Cranfield University, Cranfield, Bedfordshire MK43 OAL, UK \\ ${ }^{\mathrm{b}}$ Enhanced Composites and Structures Centre, Manufacturing and Materials Department, School of Applied Sciences, Cranfield University, Cranfield, \\ Bedfordshire MK43 OAL, UK
}

\section{A R T I C L E I N F O}

\section{Article history}

Available online 17 September 2016

\section{Keywords:}

Feed-forward observer

Error stability analysis

Intermittent fault detection

Adaptive threshold design

Mass-spring-damper system

\begin{abstract}
A B S T R A C T
This paper provided an approach to design feed-forward observer for nonlinear systems with Lipchitz nonlinearity and bounded unknown inputs (disturbances/uncertainties) to ensure the sensitivity against intermittent faults. The proposed observer design guarantees the system error stability. Some variables and scalars are also introduced to design observer's parameters, which bring more degrees of flexibility available to the designer. The designed observer is used to propose a precision fault detection scheme including adaptive threshold design to detect intermittent faults. The efficiency of the considered approach is examined by the intermittent failure case in the suspension system of a vehicle. Simulation results show that the accurate state estimation and fault detection are achieved successfully.

(C) 2016 This is an open access article under the CC BY license (http://creativecommons.org/licenses/by/
\end{abstract}

\section{Introduction}

A fault within a system is defined as an external input that causes the performance of a system to differ from a pre-determine behavior threshold. Faults are classify based on if they have grown gradually through out the operation of a system commonly distinctive of slow component wear (incipient fault); proceed quickly alike a step development as a consequence of a rapid damage (abrupt faults), or accrued in discrete periods associate to component degradation or unknown system synergy (intermittent faults). Intermittent faults can happen in all systems, mechanical, hydraulic or electronic, in an erratic manner, and if neglected over time they may emerge into severe and persistent faults. The assumed uncertainty of an intermittent fault means that it cannot be simply predicted, detected nor is it naturally repeatable during maintenance examination. Accordingly, faults of this type boost many interests in the field of through-life engineering of products [1]. Nonetheless, an intermittent fault, which is hidden in the time of regulated maintenance testing, may recur at any time eventually. Hence, the intermittent fault acts an ever-increasing dispute in the maintenance of electronic, mechanical and hydraulic equipment. An ample fraction of breakdowns associated to the intermittent faults will test well and will be classified as "No Fault Found" (NFF) [1-3].

\footnotetext{
* Corresponding author.

E-mail addresses: t.sedighi@cranfield.ac.uk (T. Sedighi), p.d.foote@cranfield.ac.uk (P.D. Foote), p.sydor@cranfield.ac.uk (P. Sydor).
}

The intermittent fault often gets worse with time, as far as it finally becomes noticeable enough to be detected with common test equipment's [4]. Hence, establishing the capacity for early detection and isolation of the intermittent fault may help to reduce extensive system damages [5].

Faults can arise in the actuators, process components or sensors. Sensor faults are of specific attention. The brunt of faulty sensors may consequence system fails to achieve its performance or cause a destructive mechanical failure [6,7].

For decades, several approaches have been introduced for detecting the feasible problems in dynamic systems to promise the common performance of the system. In routine, the designer chooses one among various fault detection (FD) approaches, according to the specification of the system and the type of possible faults. Some approaches are more appropriate for off-line FD analysis. Subspace-based detection method is one example which is introduced in $[8,9]$ which is obtained for health monitoring of mechanical structures, such as bridges. Other approaches are used for online fault detection. Model/observerbased FD approach is one of the interesting methods between the online fault detection approaches. In observer-based FD techniques generally, it is needed to design an observer that will be robust to the unknown inputs affecting the system, but tense to the faults. Next, the residual is designed according to the observer's output. Then the faults can be detect by contrasting the residual with a fixed or adaptive threshold [10].

This technique which flags the fault by inspecting the residual is named residual-based FD. However, developing standardized design techniques for systems dealing with the unknown 
disturbances and model uncertainties has been confirmed to be challenging [11-13]). When both the unknown inputs and faults affecting the residual developed by the observer-based FD, a few small faults may not be detected for the designed threshold. The intention of an ideal observer-based FD method is to reduce the faults size which are not detectable. The idea following this principle is that the designed observer should be robust to the unknown inputs/disturbances but sensitive to the faults [14,15]. In this paper, the existing approach to nonlinear observer design for the broad class of nonlinear systems is extended, which are dealing with the bounded unmatched unknown inputs (uncertainties) and experiencing sensor faults. The nonlinearity satisfies the Lipschitz condition. This method affords a direct approach for designing the nonlinear observer, which can decrease the state estimation errors against the unmatched unknown inputs (uncertainties). Then the robust FD issue of the system under the investigation is studied when a nonlinear observer was designed, and sufficient conditions are provided to make the observers asymptotically stable.

This paper is coordinated in this way: Section 2 provides the mathematical description of the nonlinear system under investigation. The design of the nonlinear observer along with theorems and error dynamic stability analysis are presented in Sections 3 and 4 respectively. In Section 5 the fault detection filter and appropriate adaptive thresholds are designed although the numerical example and simulation results are presented in Section 6. Finally, the conclusions are provided in Section 7.

\section{System description}

The state-space form of the nonlinear systems under the investigation are presented as,

$\dot{x}(t)=h_{x}\left(x, u, \mu, f_{i}\right)$,

$y(t)=h_{y}\left(x, f_{i_{s}}\right)$.

If the nonlinear function $\dot{x}(t)=h_{x}\left(x, u, \mu, f_{i}\right)$ is differentiable with respect to $x$, then this class of the system may be expressed in terms of a linear unforced part, and nonlinear state dependent controlled part [16],

$\dot{x}(t)=A x(t)+B u(t)+D \mu(t)+H S g(x, u, t)+K_{i} f_{i}(t)$,

$y(t)=C x(t)+K_{s} f_{i_{s}}(t)$,

where $x \in \mathbb{R}^{n}, u \in \mathbb{R}^{m}$ and $y \in \mathbb{R}^{p}$ present state, input and output vectors respectively. $A \in \mathbb{R}^{n \times n}, B \in \mathbb{R}^{n \times m}, C \in \mathbb{R}^{p \times n}, H \in \mathbb{R}^{n \times n}, S \in \mathbb{R}^{n \times s}$, $K_{i} \in \mathbb{R}^{n \times r_{i}}$ and $K_{s} \in \mathbb{R}^{n \times r_{s}}$ are known matrices while $f_{i} \in \mathbb{R}^{r_{i}}$ and $f_{i_{s}} \in \mathbb{R}^{r_{s}}$ are the actuator/component and sensor faults correspondingly. $D \in \mathbb{R}^{n \times q}$ is referred to the known distribution matrix of the unknown input (uncertainty), and $\mu \in \mathbb{R}^{q}$ is an unknown bounded vector which describes the unknown input and/or any kind of modeling uncertainty such as noise, time-varying term, and parameter variation. $g(x, u, t) \in \mathbb{R}^{h}$ represents Lipschitz nonlinearity in the system. The function $f(x)$ satisfies a Lipschitz condition on the interval $[a, b]$ if there exists a constant $\kappa$ (dependent on both $f(x)$ and the interval) such that $|f(x)-f(\hat{x})| \leq \kappa|x-\hat{x}|$, see [17].

Prior to nonlinear observer design, the following assumptions are made:

\section{Assumption 1.}

- The pair $(A, C)$ is observable.

- Nonlinearity $g(x, u, t)$ is assumed to be globally Lipschitz in $x$ with Lipschitz constant $\kappa$, i.e.

$\|g(x, u, t)-g(\hat{x}, u, t)\| \leq \kappa\|x-\hat{x}\|$.

- Measurement depends directly on one of the states, which is affected by the unknown input (uncertainty), $C D \neq 0$.
- The disturbance is bounded to some positive constant $\alpha$, $\|\mu(t)\| \leq \alpha$.

\section{Nonlinear feed-forward observer design}

The nonlinear unknown input observer usually has been applied to the class of nonlinear systems where $H D=0$. In this section, an observer is designed when this condition is not satisfied. In another hand the following condition is satisfied,

$H D \neq 0$.

The nonlinear feed-forward observer is designed such that its state estimation error vector $e_{x}(t)$ approaches to zero asymptotically, regardless of the presence of the unknown input term in the system. Hence the observer of the following form is given

$\dot{z}(t)=N z(t)+L y(t)+G u(t)+H^{*} S g(\hat{x}, u, t)+\Omega v$,

$\hat{x}(t)=z(t)-E y(t)$,

where $z \in \mathbb{R}^{n}$ is the state observer, with matrices $N \in \mathbb{R}^{n \times n}, L \in \mathbb{R}^{n \times p}$, $G \in \mathbb{R}^{n \times m}, H \in \mathbb{R}^{n \times n}$, and $E \in \mathbb{R}^{n \times p}$ which should be obtain. The $\hat{x}$ is an estimate of $x . \Omega \in \mathbb{R}^{n \times m}$ is the feed-forward injection map and $v \in \mathbb{R}^{m}$ is an external feed-forward compensation signal. Note that $\Omega$ is selected such as $C \Omega$ to be a nonsingular matrix. It is desired to design the observer such that $\hat{x}$ tends to $x$ eventually.

Hence the state estimation error equation of the form

$e_{x}(t)=x(t)-\hat{x}(t)=x(t)-z(t)+E y(t)$

is defined for the system (1) and observer (5) in terms of $x(t)$.

It is easy to verify that $H=H^{*}$ and rewritten Eq. (6) as follows:

$$
\begin{aligned}
e_{\dot{x}}(t)= & N e_{x}(t)+(H A-N H-L C) x(t)+(H B-G) u(t)+H D \mu(t) \\
& +H S(g(t, u, x)-g(\hat{x}, u, t))-\Omega v+H K_{i} f_{i}(t)-L E K_{s} f_{i_{s}} \\
& +E K_{s} f_{i_{s}},
\end{aligned}
$$

where

$H=I_{n}-E C$.

If the following conditions hold:

$H D \neq 0$,

$H B-G=0$

$H A-N H-L C=0$,

then the error equation (7) will find the following form:

$e_{\dot{x}}(t)=N e_{x}(t)+H S(g(x, u, t)-g(\hat{x}, u, t))+H D \mu(t)-\Omega v$.

Note that to analyze the error stability the error estimation equation should be perform in fault-free case, where $f_{i}(t)=f_{i_{s}}(t)=0$.

Eq. (12) shows that the error, $e_{x}(t)$, is sensitive to both unknown input and the nonlinearity.

To design the nonlinear unknown input observer the following definitions and assumption are given:

a. Define the output error $e_{y}(t)$,

$e_{y}(t)=y(t)-\hat{y}(t)=C e_{x}(t)$.

b. Define $v$

$v=\Theta \frac{y(t)-\hat{y}(t)}{\|y(t)-\hat{y}(t)\|}=\Theta \frac{C e_{x}(t)}{\left\|C e_{x}(t)\right\|}$,

where $\Theta \in \mathbb{R}^{m \times m}$ is a diagonal matrix which satisfies

$\lambda_{\min }(\Theta) \geq \alpha\|F\|$,

and $F \in \mathbb{R}^{m \times m}$ will be defined later. 
c. Let $P$ be the positive symmetric definite (p.s.d.) solution of the Lyapunov equation

$N P+P N^{T}=-Q_{f}$,

where $Q_{f}$ is an arbitrary p.s.d. matrix. Hence the feed-forward injection map may be present as

$\Omega=P^{-1} C^{T} \Theta^{-1}$.

Assumption 2. Assume that there exists a matrix $F \in \mathbb{R}^{m \times m}$, such as:

$H D=\Omega F=P^{-1} C^{T} \Theta^{-1} F$.

Then in order to design the observer (5) the following steps are made:

- From the Assumption 2 and condition (8), Matrix $E$ will be obtain as:

$E=(\Omega F-D)(C D)^{+}$.

where $(C D)^{+}$is a pseudo inverse of $C D$.

- After that substitute matrix $E$ into (8) to obtain matrix $H$.

- Then by substituting $H$ into (10) matrix $G$ will be given.

- Next assume that the pair $(H A, C)$ is an observable pair and $P_{0}$ is the p.s.d. solution of the following Algebraic Riccati Equation (ARE), then the gain matrix $K$ is selected to make the matrix $N=H A-K C$ stable,

$(H A)^{T} P_{0}+P_{0}(H A)-P_{0} C^{T} R^{-1} C P_{0}=-Q_{0}$,

where $Q_{0} \in \mathbb{R}^{n \times n}$ and $R \in \mathbb{R}^{p \times p}$ are arbitrary p.s.d. matrices. Hence by selecting $K=P_{0} C^{T} R^{-1}$ the matrix $N$ will be an stable matrix.

- Once $K$ is calculated, by substituting $N, K$ and $H$ into (10) the observer gain $L$ could be achieved of the form

$L=H A E+K\left(I_{p}-C E\right)$,

where $I_{p} \in \mathbb{R}^{p \times p}$ indicates an Identity matrix of size $p$.

Eventually all the design matrices for constructing the observer (5) are obtained.

\section{Stability analysis of the error system}

In this section, the behavior of the error system (7) in the faultfree case is studied. To analyze the error stability the following theorem is defined:

Theorem 1. Assume that the conditions (8)-(11) are satisfied and there is no fault in the system, then the error system (12) is asymptotically stable if the following Matrix Inequality (MI)

$$
P_{1} N+N^{T} P_{1}+\epsilon_{1} P_{1}^{2}+\frac{\kappa^{2}}{\epsilon_{1}} S^{T} H^{T} H S<0
$$

for some positive number $\epsilon_{1}$, has a p.s.d. solution $P_{1}$.

Corollary 1. If $P_{1}$ is a solution of the following ARE

$N^{T} P_{1}+P_{1} N+\epsilon_{1} P_{1}^{2}+\frac{\kappa^{2}}{\epsilon_{1}} \sigma_{M}(H S)=-Q_{\Theta}$,

where $Q_{\Theta}$ is the appropriate p.s.d. matrix and $\sigma_{M}(H S)$ is the maximum singular values of the corresponding matrix respectively. Then the error system (12) is asymptotically stable.

Note that for simplicity the index $x$ will be omitted from $e_{x}$.
Proof. Consider the following Lyapunov equation

$V=e(t)^{T} P_{1} e(t)$,

where $P_{1}$ is a p.s.d. matrix. The time-derivative of Lyapunov equation (24) is

$$
\begin{aligned}
\dot{V} & =e(t)^{T} P_{1} \dot{e}(t)+\dot{e}(t)^{T} P_{1} e(t)=e(t)^{T}\left(N^{T} P_{1}+P_{1} N\right) e(t) \\
& +(g(x, u, t)-g(\hat{x}, u, t))^{T} S^{T} H^{T} P_{1} e(t)+e(t)^{T} P_{1} H S(g(x, u, t) \\
& -g(\hat{x}, u, t))+2 e(t)^{T} H D \mu(t)-2 e(t)^{T} P_{1} \Omega v .
\end{aligned}
$$

Since for any matrices $X, Y$ and any positive number $\epsilon>0$ the following inequality satisfies

$\epsilon\left(\frac{1}{\epsilon} X-Y\right)^{T}\left(\frac{1}{\epsilon} X-Y\right) \geq 0$

then

$X^{T} Y+Y^{T} X \leq \frac{1}{\epsilon} X^{T} X+\epsilon Y^{T} Y, \quad \forall \epsilon>0$

hence for any $\epsilon_{1}>0$,

$$
\begin{aligned}
e(t) & { }^{T} P_{1} H S(g(x, u, t)-g(\hat{x}, u, t))+(g(x, u, t)-g(\hat{x}, u, t))^{T} S^{T} H^{T} P_{1} e(t) \\
\leq & \epsilon_{1} e(t)^{T} P_{1} P_{1} e(t)+\frac{1}{\epsilon} H S S^{T} H^{T} \|\left(g(x, u, t)-g(\hat{x}, u, t) \|^{2}\right. \\
& \leq \epsilon_{1} e(t)^{T} P_{1} P_{1} e(t)+\frac{\kappa^{2}}{\epsilon_{1}} H S S^{T} H^{T}\|x(t)-\hat{x}(t)\|^{2} \\
& =\left(\epsilon_{1} P_{1}^{2}+\frac{\kappa^{2}}{\epsilon_{1}} H S S^{T} H^{T}\right)\|e(t)\|^{2} .
\end{aligned}
$$

Then substituting (14), (17), (18) and (26) into (25),

$$
\begin{aligned}
V \dot{(t)} & \leq e(t)^{T}\left[\left(N^{T} P_{1}+P_{1} N\right)+\epsilon_{1} P_{1}^{2}+\frac{\kappa^{2}}{\epsilon_{1}} \sigma_{M}(H S)\right] e(t) \\
& +2 e(t)^{T} C^{T} \Theta^{-1} F \mu(t)-2 e(t)^{T} C^{T} \frac{C e(t)}{\|C e(t)\|} \leq-e(t)^{T} Q_{\Theta} e(t) \\
& +2\left\|e(t)^{T} C^{T}\right\|\left(\frac{\alpha_{1}\|F\|}{\lambda_{\min }(\Theta)}-1\right) \leq-\lambda_{\min }\left(Q_{\Theta}\right)\|e(t)\|^{2},
\end{aligned}
$$

which implies that (27) is negative definite. Therefore the error system (12) in fault free case is asymptotically stable if there exists a p.s.d. $P_{1}$ satisfying (22).

\section{Feed-forward observer-based intermittent fault detection}

Intermittent fault is described as one which endures in a system for a limited time, after which a system regain from fault and is capable to perform its intended functions without undergoing any corrective actions. Intermittent faults are difficult to diagnose and often are recurrent. An example can be an electronic circuit with loose solder joints subjected to vibration that causes intermittent fault (open circuit). In literature such type of faults is often described as no fault found (NFF) phenomena. Other names used across the literature include: erroneous removal (ER), no problem found (NPF), cannot duplicate (CND), and re-test is OK (RTOK). Such faults (discontinuities) start with very short in duration and mostly of low amplitude. Therefore, in order to diagnose such faults, an on-line and, in many cases, real-time diagnostics system is required $[18,19]$. In another hand the intermittent fault is concerned with the dynamic behavior of discrete events and reset events continuously along any path of the system's evolution. Despite the persistent fault while they appears they do not disappears and cause a major breakdown to the system's operation or the transient faults whose appears at some point of the system's operation and will disappear before any serious damage happen and is very less likely to happen again, the intermittent faults will 
appear as a set of discrete intervals which may get longer in duration and larger in amplitude until it may eventually turns to a persistent fault if it leaves unattended.

Hence, mathematically the intermittent fault could be modeled as a combination of impulses at discrete intervals:

$f_{i_{s}}(t)= \begin{cases}d d_{1} & \text { for } t<t_{1} \\ d d_{2} & \text { for } t_{1} \leq t<t_{2} \\ d d_{3} & \text { for } t_{2} \leq t<t_{3} \\ d d_{4} & \text { for } t_{3} \leq t<t_{4} \\ \vdots & \\ d d_{n-1} & \text { for } t_{n-2} \leq t<t_{n-1} \\ d d_{n} & \text { for } t_{n-1} \leq t<t_{n}\end{cases}$

where $d d_{i} \geq 0$, for $(i=1, \ldots, n)$ are constants and $t$ indicates the time.

In the real world the intervals between the faults, the duration of each interval and the amplitudes are unknown like any other faults. Hence if the amplitudes or intervals change it would not change the type of fault, unless it becomes very large in amplitude and long enough in duration to cause serious breakdown to the system operation. In this case, the intermittent fault will change to the persistent fault.

In our example system to show the better applicability of the proposed method, the intermittent fault mathematically has been injected into the system, hence the amplitude and the duration of each interval has been defined.

The intermittent fault detection system in this paper consists of two parts: residual generation and residual evaluation including thresholds.

\subsection{Residual generation}

For effective fault detection, the reaction on sensitivity due to the disturbances (unknown inputs) in the residuals should be small while that due to the faults should be large. Hence the generated residual, $r(t)$, should be as sensitive as possible to the fault, $f_{i_{s}}(t)$, and as robust as possible to the disturbance $\mu(t)$.

Consider the nonlinear system (2) with the bounded unknown disturbance (see Assumption 1). Then the fault detection filter for this system may presented as:

$$
\begin{aligned}
\dot{z}(t) & =N z(t)+L y(t)+G u(t)+H^{*} \operatorname{Sg}(\hat{x}, u, t)+\Omega v, \\
\hat{x}(t) & =z(t)-E y(t), \\
\hat{y}(t) & =C \hat{x}(t), \\
r(t) & =\xi(y(t)-\hat{y}(t))=\xi C e_{x}(t)+\xi K_{s} f_{i_{s}}(t),
\end{aligned}
$$

where $\xi \in \mathbb{R}^{n \times p}$ is the weighting matrix to be designed.

In order to design $\xi$ the following assumptions are made:

- Residual $r(t)$ is insensitive to uncertainties $\mu(t)$ (disturbance or noise), $\xi H D=0$.

- Residual $r(t)$ is insensitive to parameter errors or nonlinearities, $\xi H S=\xi \Omega=0$.

- Residual $r(t)$ is sensitive to faults, $\xi K_{s} \neq 0$.

Consequently, $\xi$ should be define to achieve the following aims [20]:

- The effect of the unknown input/disturbance signals on the residual are as small as possible while the effect of fault signal is as large as possible.

- The effect of parametric uncertainties on the residual signal are as small as possible.

- The fault detection system is robust stable in the existence of external signals and uncertainties.

The ambition is to show that when faults have arisen the residuals are vary from zero, and in the fault-free case the residual tends to zero.

\subsection{Residual evaluation}

A well-known choice of evaluation signal is the 2-norm:

$r_{\text {eval }}=\|r\|_{2} \triangleq \sqrt{\int_{0}^{\infty}|r(\tau)|^{2} d \tau}$.

Since the evaluation function (30) cannot be fulfilled accurately, because the value of $\|r\|_{2}$ is not known until $t=\infty$, and it is acceptable to consider that the faults could be detected, if they appear over finite time periods. Accordingly Eq. (30) could be changed to

$r_{\text {eval }}=\|r(t)\|_{2} \triangleq \sqrt{\int_{0}^{t}|r(\tau)|^{2} d \tau}$

where $\tau$ is the finite time window [21].

Advantage of using the 2-norm is that it is then straightforward to optimize the residual generator to minimize the impact of the unknown input, $\mu(t)$.

\subsection{Adaptive threshold}

For the evaluation signal (31), if

$r_{\text {eval }}>T_{r} \Rightarrow A$ fault is detected

and

$r_{\text {eval }} \leq T_{r} \Rightarrow$ No fault is detected.

Therefore, in no fault circumstances the value of threshold, $T_{r}$, affords a precise bound and thus shows a beneficial guidance for selecting the robust threshold [13,22].

In fault-free circumstances the adaptive threshold is constructed according to the residual dynamics. To design the adaptive threshold for nonlinear system (2) and evaluation signal (31) reformulate the residual $r(t)$ as follows:

$r(t)=r_{e}(t)+r_{f_{i_{S}}}(t)$,

where $r_{e}(t)=\left.r(t)\right|_{\mu(t)=0 . f_{i_{s}}(t)=0}$ and $r_{f_{i_{s}}}(t)=\left.r(t)\right|_{\mu(t)=0}$ are the residuals due to the error and intermittent fault correspondingly. In another hand to design $T_{r}$, one needs to define the residual due to the unknown input, $r_{\mu}(t)=\left.r(\bar{t})\right|_{f_{i_{s}}(t)=0}$ where $t=\left[0, \ldots, t_{1}, \ldots, t_{2}\right]$ and $\bar{t}=\left[t_{1}, \ldots, t_{2}\right]$. Hence, $T_{r}$ may be defined as follows:

$T_{r}=\sup \left\|r_{\mu}(\bar{t})\right\|+\left\|r_{e}(t)\right\| \geq 0$.

Since the disturbance is bounded to a positive scalar $\alpha$, then

$\sup \left\|r_{\mu}(\bar{t})\right\|=\delta_{\alpha} \geq 0$,

where $\delta_{\alpha}$ is a positive constant number. Hence $\left\|r_{e}(t)\right\|=T_{r}-\delta_{\alpha}$, which results that

$\left\|r_{e}(t)\right\| \leq T_{r}$.

Consequently to show that $T_{r}$ is the upper bound of residual $\|r(t)\|$, consider (32) as follow:

$\|r(t)\|=\left\|r_{e}(t)+r_{f_{i s}}(t)\right\|$.

In faulty case $\left\|r_{f_{i_{s}}}(t)\right\|>\beta>0$, where $\beta$ is a positive constant. Hence $\|r(t)\|=\beta+T_{r}>0$ which conclude $\|r(t)\|>T_{r}$.

If there was no fault in the system, then $\left\|r_{f_{i_{s}}}(t)\right\|=0$, therefore from (36), $\|r(t)\|=\left\|r_{e}(t)\right\|$ which results in $\|r(t)\| \leq T_{r}$.

Lastly based on the achieved outcomes the generated residual and adaptive thresholds may detect the intermittent faults while occurred. 

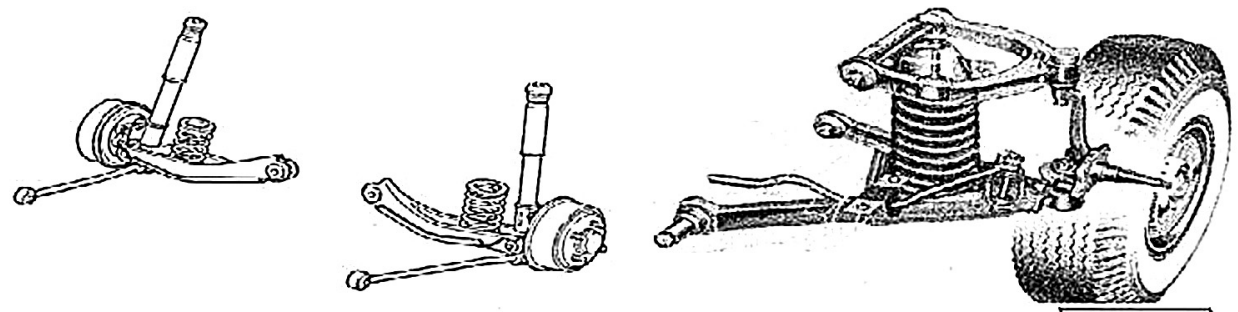

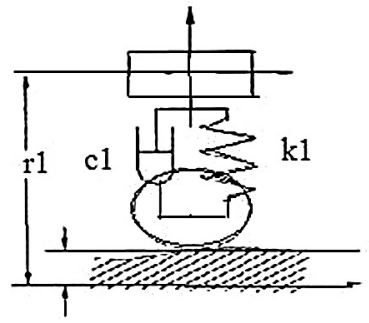

a

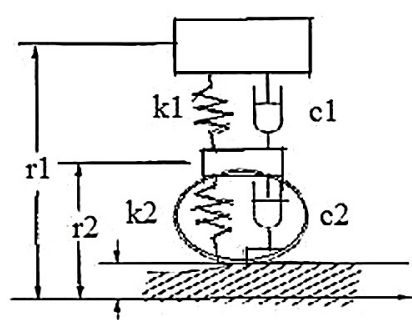

$\mathrm{b}$

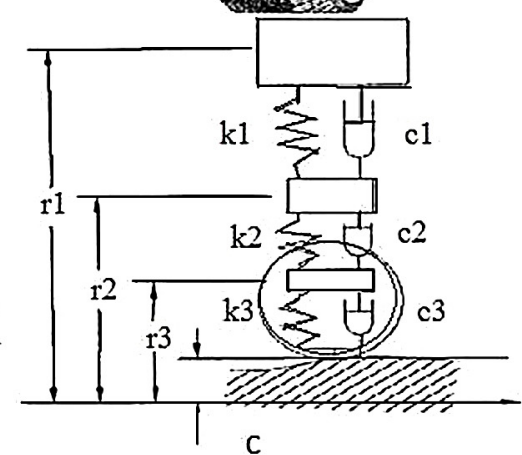

$\mathrm{C}$

Fig. 1. The model vehicle suspension system.

\section{Example system}

To demonstrate the efficiency of the outcomes achieved in Sections 2-5, consider the dynamic characteristics of a car suspension system. The system is treated as a mass-springdamper (M-S-D) system where $n$ masses, springs, and dampers are linked in series [23-25].

Since study of a full suspension model is truly complex including all four tire/suspension systems working individually, then the quarter-car suspension system may be expressed in the three levels of complexity. The one-degree of freedom model shown in Fig. 1a considers displacement $r_{1}$ of the sprung mass $m_{1}$ of the vehicle and the primary suspension stiffness $k_{1}$ and damping $c_{1}$ only. Here the unsprung mass (mass of the wheels and other components such as lower control arms) and the mass of the tires are not considered. The two degree of freedom model shown in Fig. 1b accounts for the dynamics of the unsprung mass and introduces the second equation of motion and degrees of freedom for the displacement $r_{2}$ of the unsprung mass $m_{2}$, springs and dampers with $k_{2}$ and $c_{2}$. In this model, the tires are massless. A three-degree of freedom model is shown in Fig. 1c where the dynamics of the tires are added to the analysis by treating them as a mass spring damper [26,27].

A mass-spring-damper model is normally designed by a series of differential equations. The model consists of a finite number of masses, springs, and dampers on a line. It is assumed that $n$ masses, springs and dampers are connected serially. The system which will be designed in this paper may be extended. Hence, the designer can choose any number of masses, springs and dampers to build the desired system with more complexity. In Fig. 2, two springs, dampers and masses were linked together in series [23] where $x_{1}$ and $x_{2}$ indicate the position and velocity of the first mass and $x_{3}$ and $x_{4}$ indicate the position and velocity of the second mass, respectively. $A_{n l}$ is a nonsingular damping device whose damping force is $F_{A_{n l}}=C_{n l} \operatorname{sign}\left(x_{2}\right) \ln \left(1+\left|x_{2}\right|\right)$, with $C_{n l} \geq 0$. An arbitrary and unknown force, $w$, is enforced on the second mass. The known input forces, $u_{1}$ and $u_{2}$ are applied to both masses 1 and 2, subsequently. The state variables $x_{1}$ and $x_{4}$ are measurable, hence to estimate the state variables $x_{2}$ and $x_{3}$ the observers are designed.
Assume that the state equations of the model are given such as,

$$
\begin{aligned}
& \dot{x}=\left(\begin{array}{cccc}
0 & 1 & 0 & 0 \\
-\frac{k_{1}+k_{2}}{m_{1}} & -\frac{b_{1}+b_{2}}{m_{1}} & \frac{k_{2}}{m_{1}} & \frac{b_{2}}{m_{1}} \\
0 & 0 & 0 & 1 \\
\frac{k_{2}}{m_{2}} & \frac{b_{2}}{m_{2}} & -\frac{k_{2}}{m_{2}} & -\frac{b_{2}}{m_{2}}
\end{array}\right)\left(\begin{array}{c}
x_{1} \\
x_{2} \\
x_{3} \\
x_{4}
\end{array}\right) \\
& +\left(\begin{array}{c}
0 \\
-\frac{1}{m_{1}} \\
0 \\
0
\end{array}\right) \rho\left(x_{2}\right)+\left(\begin{array}{cc}
0 & 0 \\
\frac{1}{m_{1}} & 0 \\
0 & 0 \\
0 & \frac{1}{m_{2}}
\end{array}\right)\left(\begin{array}{l}
u_{1} \\
u_{2}
\end{array}\right)+\left(\begin{array}{c}
0 \\
0 \\
0 \\
\frac{1}{m_{2}}
\end{array}\right) w
\end{aligned}
$$

$\rho\left(x_{2}\right)=C_{n l} \operatorname{sign}\left(x_{2}\right) \ln \left(1+\left|x_{2}\right|\right)$.

The output of the model is presented as, $y=C x+\eta_{y}$, while $C \in n \times n$. And $\eta_{y}$ indicates an additive offset (intermittent sensor fault/output error) on each output.

\subsection{Intermittent fault}

Collapsing suspension because of coil spring failure sounds to be a growing issue, created by a combination of latter harsh winter conditions and weight-saving designs. A plastic layer is coating the

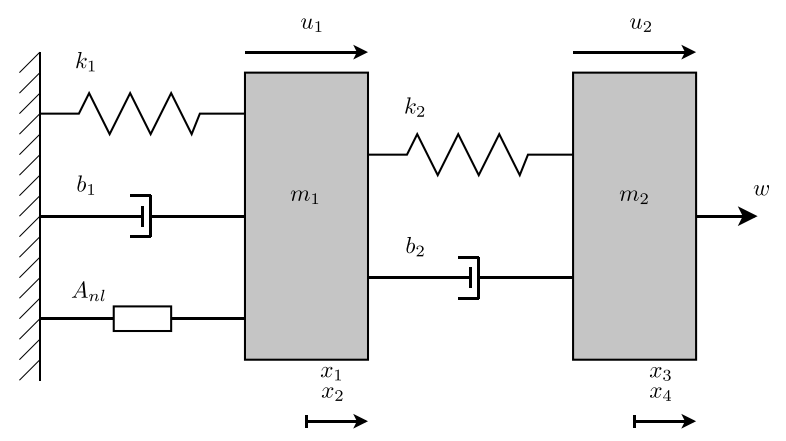

Fig. 2. The mass-spring-damper system. 
coil springs while they are build to decrease the risk of corrosion. Over time, contact between coils when the spring is compressed frequently in service may cause damage to this coating. Regularly the failure of the coil spring appears to be created by corrosion, is accelerated by salt enforced to the roads in winter. Consequently, the electrolytic action between the salt solution, created by road salting, and the iron in the spring produces free hydrogen atoms which enter the steel and can cause microscopic cracking. Cracks propagate and combine, eventually leading to the spring failure, (www.theaa.com).

Cracks and corrosion both can be classified as intermittent faults. Assume that at each failure, the length of spring will change suddenly. Hence, a fault in the position $i$, is defined as a change in the length of the $i$ th spring, $L=\left(L_{0}+f_{i_{s}} L_{0}\right)$, while in all other parts of the model the length of the springs will remain as $L=L_{0}$, where $L_{0}$ indicates the initial length of the spring.

The fault, $f_{i_{s}}(t)$ is a time varying of the form $f_{i_{s}}(t)=d d_{i} y_{n_{c}}(t)$, where the constants, $d d_{i}$, for $i=[1, \ldots, 4]$, indicate the maximum fault amplitudes. The selected output is shown by $y_{n_{c}}$ where $n_{c}=1$, $\ldots, n$ and the time is defined by $t$.

Consequently, for the model under the investigation the intermittent fault, $f_{i_{s}}(t)$, may be presented as,

$f_{i_{s}}(t)= \begin{cases}0 & \text { for } 0 \leq t<5 s \\ d d_{1} & \text { for } 5 s \leq t<7 s \\ 0 & \text { for } 7 s \leq t<11 s \\ d d_{2} & \text { for } 11 s \leq t<14 s \\ 0 & \text { for } 14 s \leq t<18 s \\ d d_{3} & \text { for } 18 s \leq t<25 s \\ 0 & \text { for } 25 s \leq t<28 s \\ d d_{4} & \text { for } 28 s \leq t<40 s\end{cases}$

with constants, $d d_{1}=0.0025, d d_{2}=0.01, d d_{3}=0.15$ and $d d_{4}=0.25$.

\subsection{Simulation results}

Assume that the model parameters have got the following values, $m_{1}=5 \mathrm{~kg}, m_{2}=1 \mathrm{~kg}, k_{1}=30 \mathrm{~N} / \mathrm{m}, k_{2}=10 \mathrm{~N} / \mathrm{m}, b_{1}=4 \mathrm{~N} \mathrm{~s} /$ $\mathrm{m}, b_{2}=2 \mathrm{~N} \mathrm{~s} / \mathrm{m}, C_{n l}=5 \mathrm{~N}$ and $w(t)=0.04 \sin (t)+2 \mathrm{~N}$, then to design the appropriate observer (5), the following matrices are obtained,

$$
\begin{aligned}
E & =\left(\begin{array}{ll}
0 & 0 \\
0 & 0 \\
0 & 0 \\
0 & -1
\end{array}\right), \quad G=\left(\begin{array}{cc}
0 & 0 \\
0.2 & 0 \\
0 & 0 \\
0 & 0
\end{array}\right), \quad H=\left(\begin{array}{llll}
1 & 0 & 0 & 0 \\
0 & 1 & 0 & 0 \\
0 & 0 & 1 & 0 \\
0 & 0 & 0 & 0
\end{array}\right), \\
L & =\left(\begin{array}{cc}
-0.3501 & 0 \\
-4.5838 & 0.4000 \\
0.2936 & 1.000 \\
-0.8632 & 0
\end{array}\right), \quad K=\left(\begin{array}{cc}
-0.3501 & 0.7234 \\
-4.5838 & -0.2351 \\
0.2936 & 1.4022 \\
-0.8632 & 0.500
\end{array}\right), \\
N & =\left(\begin{array}{cccc}
0 & 1 & 0.3501 & -0.7234 \\
-8.000 & -1.200 & 6.5836 & 0.6351 \\
0 & 0 & -0.2936 & -0.4022 \\
0 & 0 & 0.8632 & -0.5000
\end{array}\right) .
\end{aligned}
$$

From (23) the p.s.d. matrices $Q_{\Theta}$ and $P_{1}$ are obtained as follows:

$$
\begin{aligned}
Q_{\Theta} & =\left(\begin{array}{cccc}
4.7840 & -16.1760 & 1.6550 & 2.3160 \\
-16.1760 & 69.7660 & -6.2630 & -9.0150 \\
1.6550 & -6.2630 & 0.6920 & 0.9280 \\
2.3160 & -9.0150 & 0.9280 & 1.3031
\end{array}\right), \\
& \\
P_{1} & =\left(\begin{array}{cccc}
3.0828 & -2.7134 & -0.0139 & -0.4510 \\
-2.7134 & 37.7228 & -1.6128 & -1.1082 \\
-0.0139 & -1.6128 & 0.4494 & 0.5322 \\
-0.4510 & -1.1085 & 0.5322 & 2.2219
\end{array}\right)
\end{aligned}
$$

Select $\epsilon_{1}=1 \times 10^{-5}$, then $\lambda \min \left(Q_{\Theta}\right)=0.0125$, which satisfies Eq. (27) to be definite negative. Hence, the error stability of the M$\mathrm{S}$-D model is guaranteed.

Fig. 3 shows the behavior of the state errors and demonstrates that the errors between the actual and estimated states are stable and converge to zero asymptotically even though uncertainties within the system exist. Fig. 3 also shows that the designed observer satisfies the stability of the error regardless of any bounded uncertainties in absence of faults.

Figs. 4 and 5 show the residual and fixed threshold responses when the system is influenced by unknown input with the known bound. Fig. 5 shows that with a fixed threshold
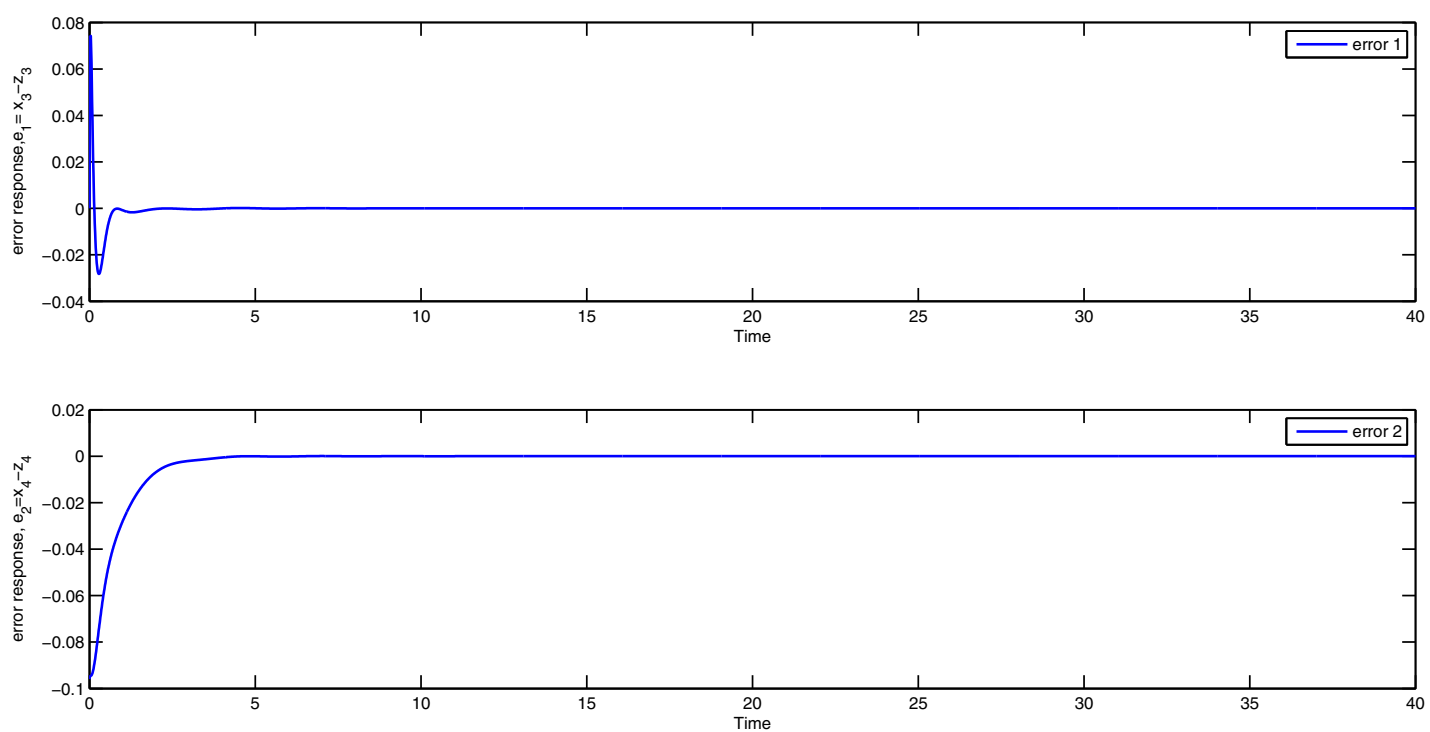

Fig. 3. The error estimation responses in presence of unknown inputs. 


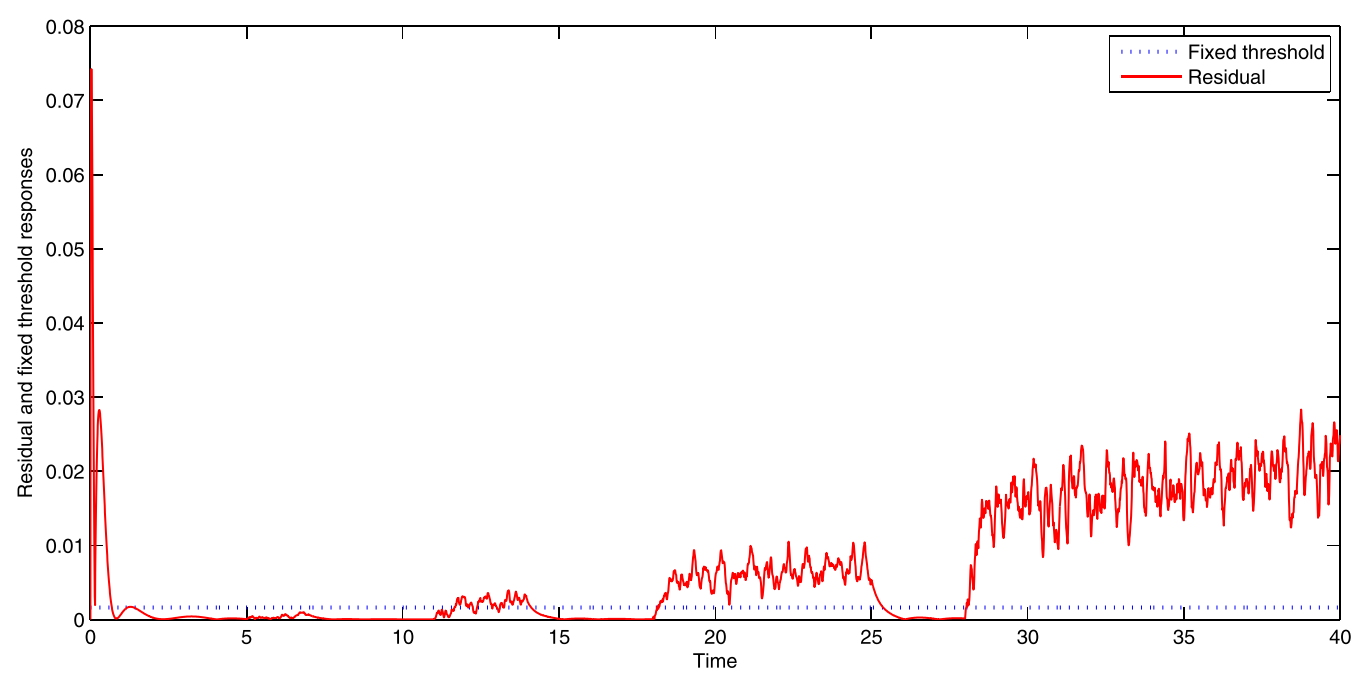

Fig. 4. The residual and fixed threshold responses in presence of the intermittent fault.

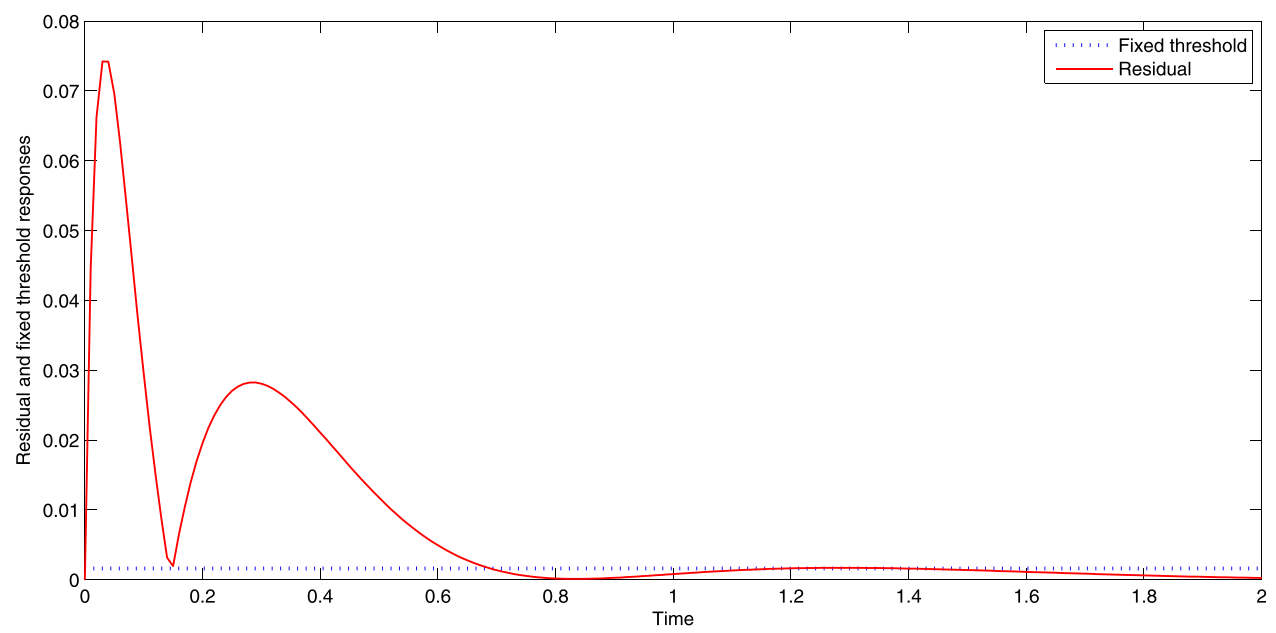

Fig. 5. The residual and fixed threshold responses in presence of the intermittent fault (first few seconds).

false alarm can occur due to the dynamics of the system. This can be seen as a breach in the fixed threshold at the beginning of the systems operation where no fault exists.

In this case, faults can be detected more precisely if an adaptive threshold is designed. When an adaptive threshold is designed, as shown in Figs. 6 and 7 the same system dynamics do not breach the threshold. With the adaptive threshold case, it is also easy to design the threshold to be insensitive to faults of specific amplitude. The adaptive threshold approach, therefore, provides the capability to ignore small intermittent disturbances that manifest as system noise and do not have a serious impact on the system operation.

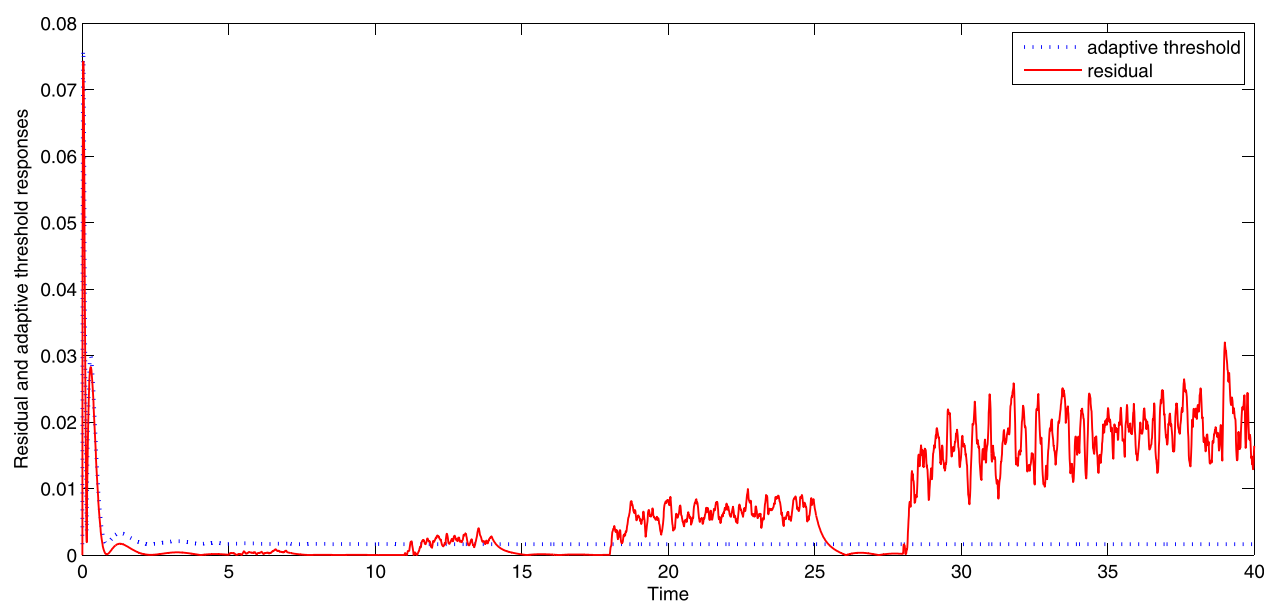

Fig. 6. The residual and adaptive threshold responses in presence of the intermittent fault. 


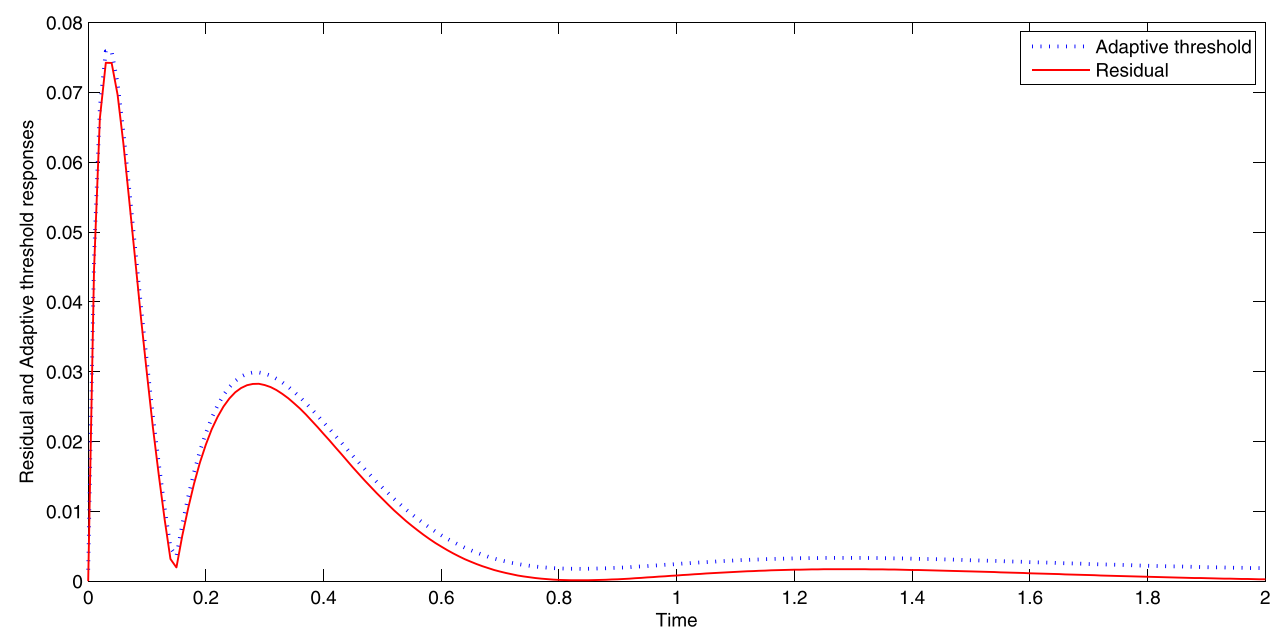

Fig. 7. The residual and adaptive threshold responses in presence of the intermittent fault (first few seconds).

The simulation results also show that the proposed design approach was minimizing the effects of any uncertainties and will give a sufficient method to design a robust observer for intermittent fault detection where the bounded disturbances exist.

\section{Conclusions}

A robust nonlinear feed-forward observer has been designed for a class of nonlinear systems whose nonlinear function satisfies Lipschitz condition, and the unknown input term is bounded. In this approach, a design matrix has been proposed to provide extra degrees of freedom to the designer to develop the residual.

The main advantage of the proposed approach is the intermittent fault detection possibility by designing a residual and an appropriate adaptive threshold while the designed threshold should be highly sensitive to the intermittent faults only.

Eventually, the effectiveness of the technique is shown by the help of a numerical example. The simulation results also demonstrate that the generated residual and adaptive threshold can indeed detect the intermittent faults regardless of the bounded disturbances/unknown inputs.

Although, there are some limitations for this work such as,

- when the intermittent faults are very small, as small as disturbance, hence they are almost impossible to detect. If the threshold has been designed to be that sensitive, then there is a possibility to detect the noises and disturbances as well,

- if the intermittent fault appears for a very short period and then goes into the rest period (deactivate period) then there is a possibility that the designed adaptive threshold was not able to detect the fault or will detect it with some delays,

but still, the proposed adaptive threshold could make the difficult intermittent fault detection an easier task for the considered class of nonlinear systems.

\section{References}

[1] Sedighi, T., Phillips, P., Foote, P.D., 2013, Model-Based Intermittency Fault Detection, 2nd International Through-Life Engineering Services Conference (UK).

[2] Hockley, C., Phillips, P., 2012, The Impact of No-Fault-Found (NFF) on ThroughLife Engineering Services, International Journal of Quality in Maintenance Engineering, 18/2: 141-153.

[3] Sedighi, T., Foote, P.D., 2015, Intermittent Fault Detection on An Experimental Aircraft Fuel Rig: Reduce the No Fault Found, IEEE International Conference on Systems and Control (Sousse, Tunisia).
[4] Steadman, B., Berghout, F., Olsen, N., 2008, Intermittent Fault Detection and Isolation System, IEEE Autotest Conference,

[5] Qi, H., Ganesan, S., Pecht, M., 2008, No Fault Found and Intermittent Failure in Electronic Products, Microelectronics Reliability, 48/66: 3-647.

[6] Sedighi, T., Foote, P.D., Khan, S., 2014, The Performance of Observer-Based Residual for Detecting Intermittent Faults: The Limitations, 3rd International Through-Life Engineering Services Conference (UK).

[7] Wang, L., Wu, L., Guan, Y., Wang, G.z., 2015, Online Sensor Fault Detection Based on An Improved Strong Tracking Filter, Sensors (Basel), 15/457: 8-4591.

[8] Basseville, M., Abdelghani, M., Benveniste, A., 2000, Subspace-Based Fault Detection Algorithms for Vibration Monitoring, Automatica, 36:101-109.

[9] Alwi, H., Edwards, C., Marcos, A., 2012, Fault Reconstruction using a LPV Sliding Mode Observer for a Class of LPV Systems, Journal of Franklin Institute, 349:510-530.

[10] Keliries, C., Polycarpou, M.M., Parisini, T., 2015, A Robust Nonlinear ObserverBased Approach for Distributed Fault Diagnosis for Process and Sensor Faults in a Class of Interconnected Input-Output Nonlinear Discrete-Time Systems, Automatica, 15:408-415.

[11] Chen, J., Patton, R.J., 1999, Robust Model-Based Fault Diagnosis For Dynamic Systems, Kluwer Academic Publishers, London, Great Britania.

[12] Chen, J., Cao, Y., Zhang, W., 2015, A Fault Detection Observer Design for LPV Systems in Finite Frequeny Domain, 88.

[13] Qning, X., Hau, Z., Feng, Y., Qiao, W.X., Yong, Y.H., 2014, Effective Model Based Fault Detection Scheme for Rudder Servo System, Journal of Central South University, 21:4172-4183.

[14] Ding, S.X., Frank, P.M., Ding, E.L., 2009, A Unified Approach to the Optimization of Fault Detection Systems, International Journal of Control and Signal processing, 14/72: 5-745.

[15] Lui, J., Wang, J., Yang, G.H., 2005, An LMI Approach to Minimum Sensitivity Analysis with Application to Fault Detection, Automatica, 41:1995-2004.

[16] Rajmani, R., 1998, Observer for Lipschitz Nonlinear Systems, IEEE Transactionas on Automatic Control, 43:397-401.

[17] Mohammad, K.m., Chadli, M., Chaabane, M., 2012, Unknown Input Observer for a Class of Nonlinear Uncertain System: An LMI Approach, International Journal on Automatic Control, 9/3: 331-336.

[18] Smith, P., Furse, C., Gunther, J., 2005, Analysis of Spread Spectrum Time Domain Reflectometry for Wire Fault Location, Sensor Journal, 5:1469-1478.

[19] Soderholm, P., 2007, A System View of the No Fault Found (NFF) Phenomenon, Reliability Engineering and System Safety, 92:1-14.

[20] Ahmadizadeh, S., Zarei, J., Karimi, H.R., 2014, A Robust Fault Detection Design for Uncertain Takagi-Sugeno Models with Unknown Inputs and Time-Varying Delays, Science Direct, 11:98-117.

[21] Puig, V., Oca, S.M., Blesa, J., 2012, Adaptive Threshold Generation in Robust Fault Detection using Interval Models: Time-Domain and Frequency-Domain Approaches, International Journal of Adaptive Control and Signal Processing,

[22] Wang, Y.Q., Ye, H., Wang, G.Z., 2007, Fault Detection of NCS Based on Eigendecomposition, Adaptive Evaluation and Adaptive Threshold, International Journal of Control, 80:1903-1911.

[23] Roch-Cozatl, E., Moreno, J., Zeitz, M., 2005, Constructive Design of Unknown Input Nonlinear Observers by Dissipativity and LMIS, IFAC, .

[24] Patton, R., Frank, P., Clark, R., 1989, Fault Diagnosis in Dynamic Systems, Theory and Applications, Prentice Hall, London.

[25] Figliola, R.S., Beasley, D.E., 2006, Theory and Design for Mechanical Measurements, 4 th ed. Wiley.

[26] Gillespie, T.D., 1992, Fundamental of Vehicle Dynamics, Society of Automative Engineer Inc.

[27] Genta, G., 1997, Motor Vehicle Dynamics: Model and Simulation, World Scientific Publishing. 